\title{
La coopération policière transfrontalière, moteur de transformations dans l'appareil de sécurité français
}

\section{Azilis Maguer}

\section{(2) OpenEdition Journals}

Édition électronique

URL : http://journals.openedition.org/conflits/901

DOI : $10.4000 /$ conflits.901

ISSN : $1777-5345$

Éditeur :

CCLS - Centre d'études sur les conflits lilberté et sécurité, L'Harmattan

Édition imprimée

Date de publication : 1 décembre 2002

ISBN : 2-7475-4291-2

ISSN : $1157-996 \mathrm{X}$

Référence électronique

Azilis Maguer, «La coopération policière transfrontalière, moteur de transformations dans l'appareil de sécurité français », Cultures \& Conflits [En ligne], 48 | hiver 2002, mis en ligne le 27 avril 2003, consulté le 30 mars 2021. URL : http://journals.openedition.org/conflits/901 ; DOl : https://doi.org/10.4000/ conflits.901

Ce document a été généré automatiquement le 30 mars 2021.

Creative Commons License 


\title{
La coopération policière transfrontalière, moteur de transformations dans l'appareil de sécurité français
}

\author{
Azilis Maguer
}

1 Le 23 août 2001, coup de tonnerre dans le ciel officiellement serein de la coopération policière transfrontalière en Europe $^{1}$ : des policiers suisses se lancent à la poursuite d'un homme qu'ils soupçonnent d'avoir volé une voiture en territoire suisse. Ils traversent la frontière franco-suisse et abattent de plusieurs balles le chauffeur poursuivi $^{2}$. Les policiers ne disposaient aucunement du droit de poursuite transfrontalière sur la base du délit supposé, ni du droit de faire usage de leurs armes à feu, n'étant pas en situation de légitime défense. Ces faits présentent sans aucun doute les traits du cauchemar qui doit agiter les responsables de la police et de la justice aux frontières intérieures des pays membres des accords de Schengen. Le développement de la coopération policière transfrontalière a pu donner l'impression initiale aux services de police frontaliers qu'ils accédaient à de nouveaux territoires d'action - au sens propre comme au sens figuré. Ces nouvelles perspectives de travail ont entraîné de nouveaux besoins - humains et techniques. Elles ont aussi entraîné la possibilité de nouveaux types d'erreurs techniques et juridiques et de fautes professionnelles et donc la nécessité de maîtriser l'usage des règles.

2 S'il est si important, dans le cas de la coopération transfrontalière européenne postSchengen, de maîtriser les pratiques, c'est aussi parce que ces dernières risquent, dans le cas d'un usage non contrôlé, d'impliquer non seulement des fautes lourdes socialement et professionnellement mais encore des atteintes de nature politique à la souveraineté de l'Etat. Rapidement, toutes les parties en présence sur le terrain ont vu les opportunités et les dangers potentiels de l'emploi de la mécanique Schengen. Tous les services - justice, police et douane - ont été placés en même temps dans cette position fort inconfortable de souhaiter - voire de devoir - employer des instruments 
caractérisés en même temps par une forte attente chez les praticiens et par un haut degré d'inflammabilité sociale et politique. C'est assurément cette situation qui a engagé les systèmes nationaux de sécurité, et dans le cas exposé ici, le système français, dans des mouvements de recomposition des rapports de travail entre les différents acteurs publics. L'impact de l'usage des règles de la coopération transfrontalière sur les rôles et sur les rapports de force entre les acteurs dépasse par ailleurs le cadre des services de coopération eux-mêmes, pour s'élargir à l'ensemble des services de la zone frontalière.

La CAAS et Mondorf, ou les effets de la gestion du travail à dix acteurs et plus

Depuis le milieu des années 1980, la coopération policière a émergé dans le discours politique public comme objet prioritaire de l'agenda européen. S'il n'est pas le mieux connu, la Convention d'Application de l'Accord de Schengen (CAAS) est du moins l'aboutissement institutionnel le plus célèbre parmi la série d'accords intergouvernementaux conclus en Europe occidentale dans les années 1990. Avant et après, à côté et à partir de ce traité, ont été conclus de nombreux accords de nature diverse qui eux aussi tentent de régler les actions de coopération, en particulier dans les zones frontalières des Etats de l'Union européenne. Ces accords fixent bien entendu des formes de coopération déjà en vigueur entre les polices frontalières, mais introduisent aussi des règles nouvelles pour l'action des services. Sur ce point, les travaux de $\mathrm{D}$. Bigo ${ }^{3}$ sur l'émergence des canaux de coopération européens des acteurs douaniers et policiers ont éclairci de larges pans de ces réseaux en formation ou en transformation dans la période d'installation du système de Schengen. Ils ont en particulier montré comment les organisations de la sécurité intérieure en Europe se constituent en « archipels", sur la base de critères d'affinités multidimensionnels, et s'autonomisent à l'égard d'autres domaines de l'action publique ainsi que des diverses sortes de contrôle. Cette modification à un niveau macrosociologique trouve des échos à un niveau de fonctionnement microsociologique. La cristallisation et la densification des réseaux et des communications entre les services en Europe ont opéré une poussée sur les rapports traditionnels entre les acteurs du champ de la sécurité intérieure.

L'impulsion institutionnelle donnée à la coopération transfrontalière transforme déjà les pratiques par le simple fait de les avaliser officiellement: il en va ainsi de la possibilité pour des services frontaliers de coopérer de manière directe sans passer par les parquets ou les autorités centrales ${ }^{4}$. Au-delà de l'officialisation de certaines pratiques, la CAAS a en outre institué de nouvelles formes d'intervention ${ }^{5}$. De la sorte, les transformations que connaît la pratique du contrôle social aux frontières intérieures des Etats de l'Union européenne relèvent d'un processus « top down » dans lequel le service local est récepteur final et applicateur de ces nouvelles règles.

5 Dans le même temps, l'acteur local ne se contente pas uniquement de son rôle de récepteur. Il investit les nouvelles formes d'action, les adapte aux contingences locales et à ses modes opératoires. Enfin, les transformations opérées au niveau local génèrent à leur tour de nouveaux besoins ainsi que des modifications dans les rapports qu'entretient l'acteur local à son organisation ou à son institution d'appartenance. De la sorte, le processus «top down » de création des normes juridiques de la coopération transfrontalière est relayé par un mouvement «bottom up » de transformation suscité au sein des appareils nationaux par les services frontaliers. 
6 La coopération transfrontalière à la frontière franco-allemande fournit un matériau intéressant pour analyser les transformations opérées au sein du système français, en raison du degré avancé d'institutionnalisation des pratiques locales de coopération.

7 Les évolutions de la coopération transfrontalière entre les polices tendent à faire de la zone frontalière un espace que tous les acteurs locaux doivent gérer en commun. La création prévue d'équipes communes d'enquête ${ }^{6}$, appelées en particulier à agir de manière transfrontalière, n'est qu'un des nombreux symptômes de cette transformation des modes de gestion de la sécurité frontalière. De la sorte, l'ensemble

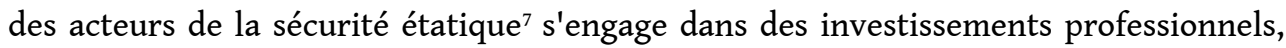
organisationnels et institutionnels nouveaux. En intervenant dans de nouveaux champs d'action, tous les acteurs frontaliers de la sécurité exercent une influence au sein de leur propre organisation, mais aussi envers leurs partenaires - anciens et nouveaux - de coopération.

8 La difficulté est alors grande de saisir l'ensemble des conséquences que ces changements induisent dans l'appareil national de la sécurité intérieure. On propose ici de la résoudre en abordant la question par le biais de la division théorique opérée par D. Monjardet ${ }^{8}$ entre les dimensions institutionnelles, professionnelles et organisationnelles de la police nationale.

9 Toutes les transformations intervenues dans les modes opératoires des acteurs locaux n'ont pas nécessairement de conséquences sur l'ensemble de l'appareil. Il ne s'agit donc pas ici de mettre au jour toutes les interactions de la coopération transfrontalière, mais de saisir celles qui ont un impact sur le système français de sécurité.

10 La mise en application de la Convention d'Application de l'Accord de Schengen (CAAS) a fourni aux services policiers et douaniers les instruments d'une coordination renforcée entre leurs modes opératoires. Avant la CAAS, la mise en place du Marché intérieur à partir du 1er janvier $1993^{\circ}$ a provoqué de profonds remaniements de la carte des services douaniers, en France comme en Allemagne. Après la CAAS, c'est l'entrée en application de l'accord franco-allemand de Mondorf-les-bains ${ }^{10}$ qui vient préciser et compléter les instruments de coordination frontalière issus de Schengen. Ces instruments fondamentaux remplacent ou complètent une multitude de structures et d'outils juridiques de coopération déjà en vigueur, et plus ou moins spécifiques à la zone frontalière ${ }^{11}$. A l'égard de ces instruments précédents, et nonobstant leur degré variable de fonctionnalité, la CAAS et Mondorf introduisent dans les pratiques de coopération un degré de formalisation et d'institutionnalisation supérieur. Par ailleurs, ces deux traités induisent la participation de tous les acteurs publics du contrôle étatique. Jusqu'à l'entrée en vigueur de ces deux accords, les relations transfrontalières et les accords qui les réglaient reposaient sur une base partenariale ne comprenant qu'un acteur public de chaque côté de la frontière. Même sans impliquer directement une coordination effective entre ces services, la CAAS et Mondorf les rendent désormais, tous au même titre, acteurs de la gestion de la sécurité dans la zone.

11 La formalisation accrue des processus de coopération, et l'implication générale, au moins théorique, de tous les services, qui l'accompagne, constituent deux facteurs déterminants des modifications intervenant de manière directe ou indirecte dans les appareils nationaux de contrôle ${ }^{12}$. Ils possèdent un poids bien plus important que d'autres éléments contenus eux aussi dans ces accords, et qui ont pu être davantage médiatisés, tels que l'observation ou la poursuite transfrontalière (Art. 40 et 41 CAAS).

Les jeux de la dimension professionnelle 
12 La CAAS prévoit que les services publics policiers et douaniers peuvent engager entre eux des actions de coopération. Ils peuvent requérir une entraide de la part de chacun des services nationaux ou régionaux désignés par les Parties contractantes. Ce point a en particulier eut l'effet, déjà noté par J. Sheptycki ${ }^{13}$, d'intégrer la gendarmerie dans les réseaux régionaux de coopération en en faisant un acteur de la coopération policière internationale. En outre, un service requérant n'est pas tenu d'adresser sa demande à un homologue professionnel. Cela signifie qu'un service douanier allemand peut demander l'entraide d'un service de gendarmerie ou de police. Les réseaux traditionnels liés à l'identité professionnelle sont donc ici fortement remis en cause.

L'accord de Mondorf-les-bains (1997) confirme et renforce cette tendance au démantèlement des réseaux locaux. Il instaure tout d'abord un Centre franco-allemand de coopération policière et douanière (CCPD) à la frontière (Offenbourg) au cours de l'année 1999. Au sein de ce Centre, des représentants des services frontaliers de contrôle ${ }^{14}$ des deux Etats coordonnent les actions de coopération et transmettent les informations demandées entre les services de la zone frontalière. L'accès direct à chacun de ces représentants permet au service requérant de choisir son partenaire, non plus en fonction d'affinités identitaires professionnelles, mais bien en fonction de la nature et du contexte de sa demande. Il n'est absolument pas tenu d'utiliser les réseaux auxquels son service recourt traditionnellement. Il peut ainsi faire appel aux compétences d'un autre service, qui seront peut-être mieux adaptées au type de sa demande. En rassemblant ainsi des personnels issus de tous les services frontaliers, le CCPD fonctionne comme une place de marché ouverte où chaque acteur est constamment susceptible de faire jouer la concurrence entre deux ou plusieurs de ses partenaires étrangers.

14 "Il y a eu un problème de chantage sur le Breisgau Milch"15. J'avais recommandé de faire passer une demande de $C R I^{16}$ à la justice française car la frontière n'est pas très loin et il était possible que le type fasse irruption du côté français. Mais ils n'avaient pas réagi, jusqu'au moment où le type fait vraiment apparition du côté français. Les Allemands sont coincés. Alors que faire? La filature opérée par les Allemands n'est pas légale, ils demandent à la police de faire partir sa $B R E C[$ Brigade régionale d'enquêtes et de coordination] pour choper le type. La Police Judiciaire a refusé parce qu'il n'y a rien de sûr et qu'elle ne veut pas. J'ai téléphoné au parquet de Colmar, le Procureur Général donne son accord à la participation des forces françaises et confie le relais à la gendarmerie. En même temps, il prévient la PJ de mon coup de fil et de l'attribution de l'affaire [au chef du service de la police nationale]. Cela a déclenché la colère [du chef du service de la police nationale], et une plainte écrite de sa part sur mon comportement incorrect. Il n'y a pas de possibilité de travail avec des réactions comme ça $»^{17}$.

Dans un système en réseau, qui fonctionne sur la base de la connaissance interpersonnelle et de l'identité professionnelle des acteurs, ce type de risque est nul ou presque. Le service qui cherche à travailler en dehors de son réseau risque de ne pas être identifié par son interlocuteur, et même de perdre sa position dans son propre réseau. Il a donc tout intérêt à continuer d'y travailler. Or, le système produit par le CCPD bouleverse ces données de l'action transfrontalière.

16 En créant entre les acteurs un accès mutuel indifférencié, le CCPD travaille à une ouverture importante des réseaux professionnels assez hermétiques établis au-dessus de la frontière. D'un côté, cette ouverture est profitable en principe à tous, puisqu'ils disposent ainsi d'une palette de services et de compétences beaucoup plus large. Les offres d'entraide sont donc plus complètes. De l'autre côté, cette extension des services 
disponibles tend à mettre en danger la stabilité des relations entre les services frontaliers. De la sorte, le service qui n'a aucun intérêt direct à une participation au $\mathrm{CCPD}^{18}$ est néanmoins tenu d'y assurer une présence.

Il en va en effet du maintien des coalitions établies entre les professions à la frontière, considérées jusque-là par les acteurs comme "naturelles». Le cadre du CCPD fonctionne comme un verre grossissant pour ce phénomène d'ouverture, qui se produit aussi entre les services frontaliers à un niveau mezzo. Pour les services, l'enjeu est alors double. Il leur faut tout d'abord assurer le maintien de leurs relations de travail avec leurs partenaires frontaliers traditionnels et veiller à ce qu'aucune des autres organisations n'engage des opérations de coopération assez importantes pour remettre en cause la qualité et la fréquence du travail réalisé dans le réseau. En même temps, les services se voient offrir des opportunités d'accès à des services situés en dehors de leurs réseaux traditionnels, qu'ils cherchent à mettre à profit. Ce double mouvement de défense des acquis et d'investissement dans de nouvelles sources d'entraide tend à créer une dynamique très forte de participation au champ de la coopération transfrontalière. Ces dynamiques sont de nature essentiellement professionnelle, puisqu'elles cherchent à mettre en valeur des intérêts et des «coalitions " propres au travail spécifique de chaque force.

Or, ces dynamiques engagent les services dans des investissements qui ne sont pas toujours nécessaires pour la réalisation des buts de leur institution. Ainsi, les douanes allemande et française n'ont été intégrées au montage du CCPD que de manière auxiliaire, dans la mesure où elles n'ont jamais manifesté un intérêt très fort à l'égard de la structure. Elles disposent de leurs propres instruments de coopération internationale, sont sourcilleuses à l'égard de la coopération avec des types d'organisations autres que douaniers ${ }^{19}$, et le niveau local ou régional de Mondorf ne correspond pas à leurs niveaux opératoires stratégiques. Dans la pratique, cette position est reflétée par la remarquable faiblesse des flux de demandes entre les représentants des deux organisations douanières au CCPD. Pourtant, elles y ont des représentants ${ }^{20}$. Présents en raison des dynamiques nouvelles intervenues dans le champ, ces fonctionnaires ont davantage un rôle de veille et de défense des intérêts professionnels que de réalisation d'objectifs opérationnels précis.

Le système Mondorf de la coopération transfrontalière pousse ainsi les logiques d'action davantage vers la défense des intérêts professionnels que vers l'accomplissement des buts de l'institution. Cet effacement des distinctions instrumentales entre les services frontaliers en faveur d'un marquage plus net des rôles et des intérêts professionnels est aussi relevé dans les relations entre les parquets et les services de police judiciaire locaux.

20 Le système transfrontalier mis en place par Mondorf est essentiellement de nature policière. A un niveau structurel, il laisse inchangé les attributions et les positions des parquets nationaux, mais ceux-ci demeurent en même temps extérieurs au fonctionnement du CCPD. Cette position extérieure et un peu en retrait résulte plus généralement d'un retard de développement des relations transfrontalières entre parquets par rapport aux relations entre services de police. Cette situation des services judiciaires dans la configuration transfrontalière locale les rend dans certains cas très dépendants des capacités de coopération et de communication détenus par les services locaux de police. Avant la mise en place du CCPD, les procureurs faisaient largement appel aux réseaux policiers pour entretenir les contacts et travailler avec leurs 
homologues frontaliers. Après l'installation du centre de coopération, ils ont recours à ce nouveau pôle de contact dont ils ne connaissent pas le fonctionnement interne. A un niveau juridique, l'application des règles de l'entraide judiciaire internationale inscrite en particulier dans la CAAS et Mondorf-les-bains a nécessité une coordination et une coopération étroite entre les parquets et les services de police chargés de les appliquer. Le travail de préparation d'une demande d'entraide judiciaire internationale ou de sa réponse nécessite souvent une maîtrise fine des divers textes de la coopération internationale, que les officiers de police judiciaire qui travaillent depuis un certain temps dans le champ possèdent à un degré parfois aussi élevé que les magistrats spécialisés. La position d'exécutant qui caractérise l'OPJ à l'égard du procureur ou du juge d'instruction est remplacée par un rôle de conseil et de coordinateur des relations transfrontalières entre services judiciaires ${ }^{21}$. Dans la relation qui lie les procureurs français à leurs OPJ gendarmes et policiers, c'est la nécessité de réaliser une opération de technique juridique sans faute qui prend le pas sur le respect des positions institutionnelles. Cette configuration offre aux OPJ une place nouvelle dans ces relations. La qualité de ce déplacement tient dans la mise en valeur de rôles et de positions professionnels, qui s'effectue aux dépens des rôles institutionnels des deux types d'acteurs ${ }^{22}$. Ici comme dans le premier cas d'interaction, la fonction d'instrument de chaque acteur à l'égard de l'autre, déterminée par leurs institutions respectives, laisse la place à une interaction qui met en valeur le savoir-faire professionnel de chacun.

21 Au-delà de ce déplacement de position de l'OPJ et du procureur, le renforcement de l'identité de l'OPJ comme spécialiste du traitement juridique des demandes de coopération policière et judiciaire complexes place le policier ou le gendarme dans l'interface de la coopération directe avec son homologue allemand. Or, dans le cas où la demande est impossible à satisfaire, c'est souvent l'OPJ qui se trouve sur le front de la discussion et qui devra justifier la raison du refus. Cette position délicate donne lieu à des stratégies de couverture - c'est le procureur qui décide - et d'explication des conflits qui bien souvent se détachent de l'argumentation purement juridique pour bifurquer vers des explications de nature culturelle.

"Il y a un problème de coordination des horaires, qui amène des problèmes car les Allemands veulent toujours avoir raison et ne veulent pas comprendre et s'adapter aux manières françaises. Pour le droit, c'est la même chose, ils sont convaincus qu'ils ont raison. Un procureur ou un juge en France, c'est une personnalité, et on ne peut pas lui faire faire ce que l'on veut. Mais ils ne peuvent pas piger. C'est comme la semaine allemande, le vendredi, il n'y a plus de criminalité. Pour la demande d'info ADN, [un policier allemand] m'a demandé hier et m'a dit qu'il avait déjà informé le procureur qu'il recevrait une demande du juge d'instruction. Or, c'est faux, ça ne peut arriver que quand le juge a rédigé et envoyé sa demande de commission rogatoire, et ça dépend de son temps pour cela. Ça peut mettre quelque temps. Pour une photocopie de photo, on m'a fait trois fois la même demande, et j'ai trois fois répondu que le poste de Soultz (Haut-Rhin) n'avait rien de meilleur en qualité. Mais ils ne veulent pas comprendre, tout simplement. On ne peut rien faire. Ok, ici, on est en Allemagne, mais il faut quand même respecter notre culture et notre manière de travailler $" 23$.

Cette capacité de l'action policière à imposer à ses dimensions institutionnelles des dimensions plus proprement professionnelles n'est pas à mettre au compte de la seule pertinence des valeurs professionnelles pour le fonctionnement du champ. Elle relève aussi de la qualité de la dynamique établie entre l'institution et le niveau local d'action 
de ses services. Or, les formes d'évolution de la coopération transfrontalière travaillent ainsi à une mise en sommeil des dimensions institutionnelles de l'action policière.

Les rapports du local à l'institution

Le développement pratique de la coopération policière n'a pas été accompagné de projets politiques ${ }^{24}$. Or, Walker a très bien montré le lien que la coopération policière entretient avec l'Etat et ses institutions. En particulier, il considère que le développement de la coopération policière en Europe fournit à l'institution policière un outil important pour s'adjuger de nouveaux territoires d'action et intervenir ainsi sur des champs de contrôle qui reviennent traditionnellement à d'autres institutions de $l^{\prime} E t^{25}{ }^{25}$. On pose ici l'idée que cette évolution a effectivement cours, mais qu'en outre cette transformation s'opère à partir du niveau local, et qu'ainsi l'institution est transformée plus qu'elle n'agrandit volontairement ses terrains d'action.

Bien entendu, les évolutions de la coopération transfrontalière, et notamment le renforcement et le réaménagement de sa structuration générale, sont l'expression d'une volonté politique. Les institutions seraient donc l'instrument de ce pouvoir pour imposer ces changements aux deux autres dimensions des polices. Or, au cours de la transformation de la volonté politique en une application concrète, le rôle effectif de l'institution comme instrument de cette transformation ne s'impose pas clairement. Dès le départ, le concept du CCPD n'a reçu l'assentiment d'aucune des forces de sécurité, parce qu'il venait bouleverser les schémas établis du travail transfrontalier ${ }^{26}$. Or, le système mis en place implique la participation de toutes les institutions, et un accord même minimal sur l'usage à en faire. Le cloisonnement institutionnel, d'une part, et d'autre part le poids des dimensions professionnelle et organisationnelle, ont empêché les institutions de définir un projet commun d'action dans le nouvel espace frontalier ${ }^{27}$. Cette incapacité des institutions à imposer un concept à destination de leurs services frontaliers est alors comblée par l'action de ces services. Ces derniers peuvent développer des actions de manière isolée et les imposer à l'institution comme projet d'action.

Le projet politique à la base de l'accord de Mondorf-les-bains n'a donc pas connu de phase institutionnelle. Il a été saisi rapidement par les diverses organisations, qui se sont chargées de lui donner des contenus correspondant à leurs intérêts particuliers. La capacité du politique à se servir de l'institution pour la réalisation de ses projets semble, dans le cas français, avoir échoué au moins en partie. La substitution des institutions par les organisations dans leur rôle d'orientation de l'action porte des conséquences pour le positionnement ultérieur de ces institutions à l'égard du fonctionnement du système frontalier de coopération.

Cette absence de projet institutionnel global laisse en premier lieu les représentants locaux du pouvoir étatique en marge du processus de développement de la coopération transfrontalière.

L'institution préfectorale en particulier ne réussit pas sa prise de participation dans les évolutions structurelles du champ, parce que les institutions centrales n'ont pas de projet précis commun à faire défendre dans le champ. Cette absence de projet et de rôle qui caractérise la position du préfet à l'égard de la coopération policière transfrontalière n'est pas en soi un problème. Il en devient un, en revanche, à la frontière franco-allemande : le représentant de l'Etat français doit gérer ses relations avec les institutions politico-administratives des Länder, lesquels ont la capacité légale et matérielle de développer des projets de coopération précis. Le déséquilibre entre ces 
deux types d'institutions politiques locales en charge des politiques publiques de sécurité provoque une rupture de communication entre les deux " partenaires ».

"A la visite du CCPD par E. Teufel [Ministre-Président de Bade-Wurtemberg], il n'y avait aucun visiteur du côté français, ce qui est considéré comme un affront par Belle. Mais cela doit, selon les Français, être vu autrement, parce que le préfet en France ne peut pas se déplacer sur une invitation du Ministre-Président à trois semaines ou 15 jours. Normalement, cela doit se faire comme une rencontre et pas comme une visite, car ce sont des égaux politiques, même si l'un est élu et l'autre est nommé. Le préfet n'a pas un rôle administratif mais politique en France, il est du même niveau. Mais ce n'est pas compris par les Allemands, c'est un problème pour le faire passer parce que les mentalités ne peuvent pas se changer comme cela, et en plus la coopération n'est pas forcément une priorité (policière) pour les responsables politiques. Mais que personne ne vienne de la France, c'est normal, on n'invite pas un préfet comme un subordonné, et d'autre part, il y a déjà ici la police nationale représentée par un commissaire-divisionnaire, et la gendarmerie par un colonel, c'est-à-dire qu'il pourrait en théorie avoir jusqu'à 3000 hommes sous ses ordres, cela fait des interlocuteurs tout à fait honnêtes pour le Minister-Präsident. Il n'a pas non plus besoin d'avoir une suite de représentants officiels ici. Si le centre était à Sélestat au lieu d'être à Offenbourg, le préfet viendrait bien sûr. Mais il y a des formes à respecter quand on s'adresse à des autorités politiques. Le préfet est le représentant de l'Etat, et joue un rôle plus politique que le président du conseil régional ou général par exemple. On ne peut pas les inviter en claquant du doigt $»^{28}$.

Cette institution est au mieux une tribune locale de concertation et de coordination pour les différentes organisations policières. En revanche, les services opérationnels locaux développent à leur échelle des projets très concrets qu'ils font ensuite remonter au niveau central pour les faire avaliser par leur institution respective. La place laissée vacante par les institutions policières et politiques est ainsi occupée par le niveau local des organisations. Ce niveau investit un rôle de concepteur de projets, ensuite défendus par ses institutions centrales ${ }^{29}$.

31 Les services locaux de police réussissent variablement à investir ce nouveau rôle. De même, la capacité et la disposition de l'institution centrale à fonctionner comme une chambre d'enregistrement et de défense de projets issus du local ne sont pas égales entre toutes les polices. Les capacités d'un service à faire émerger un projet institutionnel cohérent dépendent ainsi de ces deux aspects. Or, ces derniers sont déterminés en grande partie par l'organisation de la communication interne entre le local et le central. On observe ainsi que les forces de police qui disposent d'une chaîne de communication avec peu de relais offrent la plus grande réactivité à l'égard des transformations au niveau local ${ }^{30}$. La plupart des réalisations importantes de la zone frontalière a été ainsi tirée par des polices disposant d'une communication interne efficace (gendarmerie et police des Länder). La faiblesse de la structuration centrale de ces forces permet en outre de relier les services locaux à un petit nombre d'interlocuteurs centraux, lesquels disposent ainsi d'une vue d'ensemble complète sur les développements de la zone. De la sorte, l'élaboration d'un projet global d'action sur un territoire est plus aisée que pour une organisation qui, même au niveau central, ne dispose pas de cette capacité synthétique et réactive. La police nationale est ainsi, pour cette raison structurelle de séparation très forte entre ses composantes organisationnelles, incapable de développer de véritable projet institutionnel au niveau de la zone frontalière. Elle dispose en revanche d'une structure très lourde au niveau central, qui est un instrument de pression très efficace auprès du champ 
gouvernemental. Mais les décisions que cet instrument sert à entériner ne sont alors pas des projets institutionnels. Elles travaillent surtout à la défense sectorielle des intérêts de ses diverses composantes. A l'inverse, la faiblesse de l'appareil central de la gendarmerie ne lui permet pas de peser véritablement sur le processus législatif.

Le déplacement des rôles institutionnels est ainsi tout à fait observable dans la gendarmerie nationale, qui laisse à ses niveaux d'action régionaux ou locaux de larges capacités de proposition, et charge son niveau central de guider et de défendre les concepts développés. Cela signifie tout d'abord que l'institution a reconnu le niveau régional comme un niveau pertinent de développement. Cette attitude implique par ailleurs que ce niveau peut capter les demandes issues des partenaires directs de coopération et surtout qu'il peut les faire valoir au niveau central, compensant ainsi l'incapacité du centre à développer lui-même des concepts.

$C^{\prime}$ est donc du niveau local que proviennent les idées d'action que le centre retraduit en projet. Or, le niveau local ne peut proposer que si les partenaires locaux étrangers avec lesquels il travaille sont eux-mêmes dans une dynamique de développement de l'action transfrontalière. Dans le cadre d'une dynamique transfrontalière locale, les projets d'action peuvent provenir des services allemands locaux, eux-mêmes soutenus par des projets institutionnels déterminés. C'est bien le schéma d'interaction présenté à la frontière franco-allemande. La capacité de certains acteurs allemands à développer des concepts généraux d'action dans la zone transfrontalière et à les proposer à leurs partenaires français pousse ainsi le niveau local français à transmettre au niveau central des projets d'action fortement influencés par le concept stratégique allemand. De la sorte, deux canaux exercent une influence sur la configuration des projets d'action des services de police français. Le premier canal est celui de l'interaction directe entre les représentants des institutions centrales françaises et allemandes. Le second canal est celui qui passe du niveau central au niveau local pour remonter vers le niveau central du partenaire de coopération. Dans la mesure où le niveau local français ne reçoit de ses instances centrales aucune orientation précise, les projets des partenaires allemands ${ }^{31}$ n'ont pas à s'imposer à des contre-projets français. Les décisions des institutions policières françaises sont donc fortement influencées par les projets allemands de gestion de la frontière commune. Même dans les cas où un service français prend une décision d'alliance ou de participation au champ, elle relève rarement d'une décision autonome, mais bien plutôt d'un mouvement d'adaptation aux demandes du partenaire ou aux changements que ce dernier a déjà amorcés avec d'autres forces françaises de police.

Un troisième type d'impact que l'évolution de la coopération transfrontalière à la frontière franco-allemande entraîne sur le fonctionnement des institutions policières touche à la capacité décisionnelle de ces institutions. Théoriquement, l'institution qui dispose d'un projet d'action pour ses services est capable, en fonction du contenu de ce projet, de décider aussi des modes opératoires stratégiques à adopter pour le conduire. Dans la mesure où des pans entiers de ce projet font défaut aux institutions policières, il leur manque aussi les repères nécessaires pour décider des modes d'application. L'institution tend alors à déléguer la prise de décision à l'acteur frontalier local. Ce phénomène s'observe tant à l'égard des services policiers que des parquets locaux. Cette délégation informelle du pouvoir décisionnel en matière de politique d'intervention dans le champ de la coopération crée bien entendu des effets émergents, tels que le manque d'unité entre les comportements des différents acteurs français à 
l'égard de leurs partenaires allemands. Cette forme de délégation de la capacité de décision provoque aussi chez l'acteur local un sentiment d'incertitude sur l'avenir de la mesure engagée. Ce sentiment déjà fortement présent au sein des services policiers se retrouve aussi chez les services de la justice. Dans le cas de demandes d'entraide traitées sur la base de Schengen ${ }^{32}$, et en l'absence de jurisprudence sur laquelle étayer la pratique, les parquets agissent sans disposer de guide d'action. Cela provoque des lectures différentes des textes en vigueur entre les parquets frontaliers, et des applications qui ne peuvent avoir comme contrôle que la sanction a posteriori de l'annulation de la procédure. La nécessité d'engager des actions de coopération ${ }^{33}$ et l'absence de soutien clair venant des institutions centrales renforcent la nécessité de coordination entre les acteurs locaux de la coopération et accroît encore l'écart entre les intérêts des institutions centrales et les stratégies des services frontaliers.

L'impact de la coopération transfrontalière sur les organisations

Les réalisations de la coopération transfrontalière depuis l'entrée en vigueur de la CAAS n'ont pas seulement affecté les dimensions professionnelles et institutionnelles des polices en France. La plupart du temps, les déplacements opérés sous ces deux premiers aspects ne sont pas ceux qui retiennent le plus fortement l'attention, parce qu'ils ne prennent pas des formes directement appréhensibles et qu'ils sont bien souvent progressifs. Des transformations bien plus concrètes et parfois plus radicales touchent en revanche la dimension organisationnelle de ces polices.

$\mathrm{Au}$ moins deux dynamiques locales sont à l'œuvre dans ces transformations et poussent sur les structures établies.

La première dynamique, et sans aucun doute la plus forte, est liée au phénomène d'ouverture des réseaux introduit par la CAAS et confirmé par Mondorf-les-bains. Dans ce processus d'ouverture, la zone régionale transfrontalière a gagné en pertinence pour l'action des polices. L'élaboration de ce nouvel espace d'action, la densification des structures spécifiques à une action transfrontalière, ont tiré les acteurs de la zone vers des logiques d'action régionales, et vers un recours à ces nouveaux organismes. Or, ceux-ci viennent s'ajouter à des structures de coopération opérationnelle ${ }^{34}$ propres aux organisations policières, et qui agissent à l'échelon local, régional ou central. De la sorte, les dynamiques d'action créées autour du fonctionnement du CCPD ont tendance à reporter un certain nombre de demandes d'entraide, en principe destinées à ces structures centrales, vers l'organisme régional $\mathrm{du}$ CCPD. Ce report a eu des conséquences importantes sur certaines structures qui ne disposaient pas de mécanisme de protection, comme par exemple une position de monopole dans le champ, pour se maintenir de manière certaine dans l'espace de la coopération internationale ${ }^{35}$. Au niveau central, des phénomènes de re-dynamisation de certains services de coopération sont observables dès l'entrée en fonction du centre de d'Offenbourg.

38 Ainsi, la douane française dispose de l'AAMI ${ }^{36}$, qui centralise et traite toutes les demandes d'entraide douanière à destination ou en provenance de l'international. Or, l'entrée en fonction du CCPD a correspondu à une augmentation très nette du nombre des dossiers traités par le département des relations avec l'Allemagne au sein de cet organisme centralisé. Ce mouvement d'activité a été impulsé par la crainte de ce département central de voir une grande partie des dossiers lui échapper et transiter désormais par la structure frontalière. Sa stratégie de réaction a donc été d'améliorer sa productivité, afin de rendre son service attractif et de justifier ainsi de son existence. 
D'autre part, l'organisation a souhaité défendre sa structure interne de coopération en recommandant à ses services régionaux frontaliers de ne pas recourir aux services du $\mathrm{CCPD}^{37}$. Les nouvelles conditions de l'action transfrontalière ont ainsi de larges répercussions sur l'activité d'organismes centraux.

L'impact des nouvelles structures se fait aussi sentir sur les organes de coopération placés au sein des services régionaux. Le CCPD empiète ainsi sur des domaines des services centraux, mais surtout sur les activités développées dans la décennie 1990 par les services de coopération locaux ${ }^{38}$. Ceux-ci ont réagi de manière un peu différente à l'implantation du centre de coopération à offenbourg. Explicitement menacées par le texte de l'accord de Mondorf-les-bains ${ }^{39}$, ces activités et les services qui les mènent se sont néanmoins maintenus après 1999, parce qu'ils représentaient une ressource propre aux services locaux, qu'ils rendaient ainsi en partie indépendants de l'expertise et des ressources centrales. Ces structures locales ont été conservées en réorientant fortement leur activité et/ou leur terrain d'action. C'est ainsi le cas des commissariats binationaux.

«Pour les commissariats communs, l'accord de Mondorf-les-bains prévoit leur suppression, mais l'arrangement entre les ministres de l'Intérieur de 1995 décide de la pérennité de ces commissariats. Il n'y aura donc pas de disparition, même s'ils risquent de faire double emploi $»^{40}$.

41 Le service de coopération transfrontalière au sein du SRPJ de Strasbourg, use de la même stratégie, notamment en redéployant son activité vers les Länder de la Sarre et du Rhénanie-Palatinat.

42 La seconde dynamique qui travaille à la transformation des organisations policières en France provient de l'effet de concentration de certains domaines d'action au sein du CCPD. Il rassemble en un seul organisme les missions de coordination et de transmission de l'information assurées jusque-là de manière dispersée par chaque organisation. Pour ces services, cette concentration signifie en particulier que les activités réalisées au sein de cet organisme commun ne peuvent plus former un levier efficace d'expansion fonctionnelle dans la zone. Elles sont en effet effectuées dans un cadre commun, et leur mise en valeur ou leur extension ne pourrait se faire qu'en y incluant l'ensemble des participants. La mise en valeur des compétences d'un acteur particulier est donc délicate. Cette situation pousse ainsi les acteurs locaux, non seulement à réorienter leur activité, mais aussi à investir des niches d'activité transfrontalière, souvent à la marge des opérations transfrontalières elles-mêmes, telles que l'entraide technique et la formation linguistique des personnels. Les opérations de coopération engagées en dehors du CCPD après son entrée en fonction ne touchent en effet aucun des domaines déjà investis par le centre. Par ailleurs, ces opérations ne sont pas réalisées sur le schéma du centre, en réunissant des représentants de tous les services de police, mais à nouveau sur le schéma antérieur de groupes binationaux composés de deux acteurs. Ces regroupements se font donc dans des niches d'activité, et travaillent à une spécialisation accrue des services frontaliers dans le domaine de la coopération transfrontalière. Cette spécialisation touche le personnel frontalier, mais s'opère aussi par l'élaboration de structures permanentes qui fournissent un service de coopération dans un domaine précis d'action. Ces structures amènent ainsi une division du travail de coopération de plus en plus fine au sein des services de police frontaliers. Cette division du travail de coopération luimême vient de la généralisation de la coopération comme mode d'action possible de tous les services. 
43 La spécialisation ne différencie ainsi plus seulement les services de coopération des services chargés de missions de police classiques. Elle travaille aussi à la division du travail au sein des services chargés des missions de coopération. Cette spécialisation allonge les chaînes organisationnelles et concourt à l'émergence de nouveaux métiers et de nouvelles responsabilités dans les organisations policières.

Dans le même temps, cette spécialisation de plus en plus poussée des services locaux dans la pratique de la coopération transfrontalière se nourrit d'un report sur ces acteurs locaux de certaines tâches jusque-là assumées par des services centraux. Des secteurs de la formation linguistique ont ainsi été déconcentrés et retirés des centres de formation de la gendarmerie nationale pour être insérés dans des centres francoallemands frontaliers tournés spécifiquement vers ces secteurs ${ }^{41}$. La délégation informelle de capacités décisionnelles réalisée par l'institution vers le local se double donc d'une délégation de missions de coopération qui renforce encore la pertinence de l'espace et des acteurs locaux pour gérer les activités de coopération transfrontalière.

Cet allongement des chaînes organisationnelles au sein des acteurs locaux est en partie compensé par une concentration systématique des capacités et des services spécialisés dans des structures binationales. Dans le domaine de la formation, mais aussi de la coordination des opérations de coopération ou du soutien technique ou juridique, les reports d'activité du central vers le local opèrent ainsi un déplacement vers leur lieu d'application. Cette déconcentration d'activités opère alors une re-concentration locale par la participation de services allemands à ces nouvelles activités déconcentrées. Ces dernières gagnent par ailleurs une plus-value en s'intégrant directement dans des structures binationales. Enfin, elles solidifient l'espace de coopération en le contractualisant de manière durable dans ces organismes. L'économie du travail au sein des organisations se réalise ainsi par une élaboration binationale locale des structures de coopération. Ce processus confirme le statut particulier et la différenciation des services frontaliers à l'égard des services centraux, mais aussi à l'égard des autres services territoriaux relevant de la même organisation.

La conjonction de ces mouvements de restructuration organisationnelle construit donc la spécificité de l'espace frontalier. Les nouvelles structures participent à la cristallisation de la zone transfrontalière comme espace singulier de l'action policière. L'espace et les organisations s'influencent mutuellement, amenant des modifications de l'un et des autres. Ainsi, ce processus de déconcentration, suivi d'une re-concentration locale et binationale de certaines activités, produit à son tour certains effets émergents au sein des organisations participantes, en particulier en raison du côtoiement constant d'agents issus d'organisations au fonctionnement complètement différent.

Le premier effet émergent de cette nouvelle situation interactionnelle est sans aucun doute la possibilité offerte à présent aux agents des services en coopération, et particulièrement à ceux qui sont affectés dans les structures locales binationales, d'établir un parallèle entre leurs conditions de travail - juridiques et techniques en particulier - et celles de leurs homologues étrangers. La position frontalière et la densité des rapports transfrontaliers dans la zone rapprochent tout d'abord des capacités et des modes d'action différents, dont les acteurs frontaliers tirent profit pour engager de nouvelles coopérations. La confrontation constante de deux systèmes de fonctionnement et de plusieurs polices donne la possibilité d'en exploiter les différences et de travailler à une complémentarité des pôles de compétences de chacun. Le mécanisme de formations croisées mis en place entre la gendarmerie d'Alsace et la 
police de Rhénanie-Palatinat permet ainsi de former en Allemagne, pour le compte de la gendarmerie, des chiens de cadavre ${ }^{42}$. En contrepartie, la gendarmerie forme au pistage des chiens de la police de ce Land. Des formations croisées sur la conduite automobile et motocycliste existent aussi entre ces deux partenaires. Les pratiques de coopération vont donc bien au-delà de l'intervention ponctuelle sur des cas d'infraction singuliers. Elles tendent à utiliser les pôles d'excellence des partenaires étrangers pour améliorer les capacités d'action des services dans le cadre national d'intervention. En particulier, ce type de coopération doit permettre d'améliorer la qualité du travail et des ressources d'action et ainsi la compétitivité des services à l'égard de concurrents nationaux potentiels.

Le second de ces effets émergents se manifeste par la disponibilité d'un nouvel appareil de références professionnelles. Ces capacités de référence permettent l'émergence de nouveaux jeux du local frontalier à l'égard du central au sein de chaque organisation. Les différentiels de capacités d'action notés par les services en coopération sont utilisés par ces derniers comme des moyens de pression sur le central pour l'attribution de moyens d'action à la hauteur de ceux du partenaire étranger local. La cristallisation et la formalisation des relations de coopération sous la forme de structures locales binationales engagent ainsi les organisations à un partage théoriquement égal du poids financier et technique de ces créations. Les organisations se doivent par ailleurs d'assurer à leurs services les moyens d'appliquer la politique de coopération amorcée par ces diverses créations ${ }^{43}$. Les services locaux disposent ainsi, en jouant sur ces diverses dimensions, d'un argument de pression à l'égard de leurs institutions centrales qui peut se révéler très efficace pour se faire allouer des moyens d'action supplémentaires et spécifiques ${ }^{44}$. Le développement des capacités d'action des services locaux se nourrit de la sorte de l'importance croissante donnée dans le discours et dans les faits à la pratique de la coopération transfrontalière. La coopération sert donc les services locaux autant que ceux-ci la servent.

Ces deux effets émergents sont aussi le signe d'une dynamique d'adaptation générale et progressive aux modes opératoires des partenaires de coopération. Si on se concentre ici sur les adaptations au sein des services français, ce mécanisme se laisse aussi appréhender sur les interventions des services frontaliers allemands ${ }^{45}$. Cette adaptation entre les modes d'intervention des partenaires de coopération manifeste plusieurs caractéristiques.

50 Ces phénomènes d'adaptation ne sont observables que chez des services amenés à coopérer de manière fréquente et étroite avec des partenaires frontaliers. D'autre part, cette adaptation ne peut survenir que dans les cas où elle représente un enjeu particulier pour les services en interaction. Les commissariats binationaux ${ }^{46}$ fonctionnent depuis leur création sur une organisation complètement différente de gestion des effectifs français et allemands, et il n'y a pas eu d'adaptation mutuelle pour obtenir un roulement symétrique des personnels affectés ${ }^{47}$. Il n'y avait pas non plus d'enjeu important à la réalisation d'une telle adaptation.

51 En revanche, dans le domaine technique et juridique, des efforts sensibles sont consentis par certains acteurs frontaliers. Le SRPJ de Strasbourg dispose ainsi depuis 1994 d'une Brigade régionale d'enquêtes et de coordination (BREC). Elle permet au SRPJ de coopérer avec les services d'intervention spécialisés allemands, chargés en particulier de la conduite des mesures d'observation, à un même niveau de prestation de services. Ce ne sont pas nécessairement les priorités d'action des partenaires 
étrangers qui influent directement sur les adaptations organisationnelles des services français. Plus exactement, l'influence est exercée par les priorités de la coopération avec les partenaires allemands.

Ici, la transformation de la BREC, passée de la lutte contre les violences urbaines à celle contre les trafics transnationaux, coïncide avec le besoin grandissant d'instruments de travail transfrontaliers. De la sorte, l'implantation de ce service à Strasbourg sert autant la coopération transfrontalière que les buts premiers de ce type de service.

Ces mécanismes de mise à niveau entre les modes opératoires des partenaires frontaliers se retrouvent aussi chez les services de la justice. Au niveau juridique, des adaptations ont été faites, depuis l'entrée en vigueur de la Convention d'Application de l'Accord de Schengen, entre les lectures française et allemande de certains des délits pouvant donner lieu à une poursuite transfrontalière ${ }^{48}$. C'est ainsi que le parquet de Strasbourg a reconnu comme admissible la poursuite transfrontalière sur un délit de vol à la roulotte. Cette infraction constitue en effet un vol aggravé dans la législation allemande, quand il n'est considéré que comme un vol simple en France. A la suite de plusieurs interventions transfrontalières allemandes engagées sur la base de ce délit, le parquet français a eu tendance à reprendre la qualification de vol aggravé, qui permettait ainsi de ne pas frapper d'illégalité la poursuite allemande sur le sol français. D'un autre côté, les services allemands se sont aussi adaptés aux conditions françaises, en réduisant drastiquement le nombre de poursuites transfrontalières ${ }^{49}$.

Le développement de la coopération transfrontalière n'introduit pas des bouleversements dans les systèmes nationaux. Il opère néanmoins des déplacements des rôles entre l'organisation et l'institution, entre les niveaux local et central, enfin, entre les acteurs judiciaires et policiers. La spécialisation croissante des services frontaliers tend à faire d'eux et de la zone qu'ils gèrent des éléments distincts du reste du territoire national. Les décisions stratégiques de gestion sont remises au niveau local, le niveau central n'opérant plus que comme un soutien à des logiques d'action régionalisées. La zone frontalière franco-allemande peut être considérée comme une zone expérimentale, parce qu'elle est le lieu d'application de nouvelles règles d'action, mais aussi parce qu'au cours de cette application, la confrontation directe entre deux systèmes sociaux entraîne des conflits entre les ordres politiques et culturels. En multipliant les zones de frottement - ou de friction? - entre plusieurs espaces sociaux, le développement similaire qui semble se dessiner aux autres frontières françaises ${ }^{50}$ est susceptible de renforcer les processus de transformation évoqués précédemment au sein des appareils de la sécurité et du contrôle en France.

\section{NOTES}

1. Les réflexions développées au long de cet article ont préalablement fait l'objet d'une communication présentée dans le cadre d'un séminaire du Gern (Groupe Européen de Recherches sur les Normativités, CNRS), «Questions de police/Policing Matters » 
(Paris, 23-24 Mars 2001), organisé par Jean-Marc Berlière, Clive Emsley, René Lévy et Dominique Monjardet.

2. . Le Monde du 27.08.01.

3. . Bigo D., Polices en réseaux, Presses de Sciences Po, Paris, 1996.

4. . Inscrite dans l'article 39-1 de la CAAS et dans le titre 2 de l'accord de Mondorf-lesbains (1997) sur la coopération transfrontalière franco-allemande.

5. . Telles que l'observation ou la poursuite transfrontalières.

6. . Journal officiel des Communautés européennes : Décision-cadre du conseil du 13 juin 2002 relative aux équipes communes d'enquête, 2002/465/JAI, 20.06.2002.

7. . Les services locaux étudiés sont ceux de la justice (Parquets allemands et français), de la gendarmerie départementale, de la sécurité publique, de la police aux frontières, de la police judiciaire et de la douane travaillant en coopération avec les services allemands frontaliers (police du Bade-Wurtemberg, du Rhénanie-Palatinat et de la Sarre, Service fédéral de protection des frontières - BGS - et douane).

8. . Monjardet D., Ce que fait la police, Paris, La Découverte, 1996, p.199.

9. . Instauré par le traité de Maastricht.

10. . Journal Officiel de la République française : Décret $n^{\circ} 2000-924$ du 18 septembre 2000 portant publication de l'accord entre le gouvernement de la République française et le gouvernement de la République fédérale d'Allemagne relatif à la coopération dans leurs zones frontalières entre les autorités de police et les autorités douanières (ensemble une déclaration), signé à Mondorf-les-Bains le 9 octobre 1997, pp. 14967-14972.

11. . Tels que les Bureaux à Contrôles Nationaux Juxtaposés, structures binationales douanières, et les Commissariats binationaux, structures gérées par la police aux frontières française et le service fédéral de protection des frontières allemand. 12. . Sur la capacité concrète des services à introduire les nouvelles règles d'action dans leur pratique, cf. Alain M., « Les heurts et les bonheurs de la coopération policière internationale en Europe, entre la myopie des bureaucrates et la sclérose culturelle policière ", Déviance et Société, n³, septembre 2000, volume 24, pp. 237-253. 13. . Scheptycki J.W., «Faire la police dans la Manche : L'évolution de la coopération transfrontalière (1968-1996) », Cultures \& Conflits, Paris, L'Harmattan, n²6-27, hiver 2001.

14. . Ce sont des agents issus de la Police Aux Frontières, de la Police Judiciaire et de la Sécurité Publique (Police Nationale), de la gendarmerie et de la douane française, ainsi que des Polices de deux des trois Länder frontaliers (Bade-Wurtemberg et RhénaniePalatinat), du Service fédéral de protection des frontières (BGS) et de la douane allemands.

15. . Entreprise laitière régionale allemande.

16. . Commission rogatoire internationale.

17. . Entretien avec un policier allemand au Centre franco-allemand de coopération policière et douanière à Offenbourg, le 19 avril 2000.

18. . Par exemple, parce que le type de demande qui y est traitée ne correspond pas à son profil opérationnel. C'est le cas pour les douanes, tant française qu'allemande.

19. . Dans le même sens, cf. Bigo D., Polices en réseaux, op.cit., p. 194.

20. . Deux pour le service allemand et cinq pour le service français.

21. . Même sans se placer dans le contexte particulier de la coopération transfrontalière, il a été démontré que la position institutionnelle de l'OPJ à l'égard du magistrat ne correspond pas à la pratique des rapports entre ces deux acteurs. Voir en 
particulier Mouhanna C., Polices judiciaires et magistrats, une affaire de confiance, La Documentation française, Paris, 2001, 251 p. Cet écart est seulement encore plus net dans la pratique de la coopération transfrontalière, en particulier en raison de l'introduction simultanée de nombreuses règles nouvelles de l'action policière.

22. . Par exemple, le rôle de contrôle de l'institution judiciaire sur l'action policière est fortement remis en question dans les relations entre le magistrat et le policier opérant au sein du CCPD. Par ailleurs, le rôle d'exécution de l'OPJ est nuancé par sa capacité à influer sur le travail propre au magistrat - ainsi, dans la présentation des commissions rogatoires internationales et l'utilisation des références aux règles de droit pénal disponibles.

23. . Entretien au Centre franco-allemand de coopération policière et douanière d'Offenbourg avec un gendarme, le 14 avril 2000.

24. . Walker N., « European Integration and European Policing », 23 p., in Anderson M., Policing across National Boundaries, Londres, Pinter Publishers, pp. 23-24.

25. . Ibid., p. 26.

26. . A l'exception sans doute de la police aux frontières, porteuse avec son partenaire allemand du service fédéral de protection des frontières, du projet initial et, à la marge, de la gendarmerie, qui avait cependant ses propres structures de coopération à défendre.

27. . Walker N., « The New frontiers of European Policing », pp. 165-186, in Anderson et al., The Frontiers of Europe, Londres, Pinter Publishers, 1998, $260 \mathrm{p}$.

28. . Entretien au Centre franco-allemand de Coopération policière et douanière à offenbourg avec un gendarme, le 11 mai 2000.

29. . L'accord de Metz de 1992 entre les trois Länder frontaliers et les services de police des trois départements frontaliers, signé entre le préfet de la région lorraine et les ministres de l'Intérieur des trois Länder, met ainsi en place des cellules de coopération au sein des services régionaux de police judiciaire et des groupes de travail binationaux. De même, le projet de convention franco-allemande sur la coopération entre les services de sécurité fluviale a été largement élaboré par les services locaux concernés.

30. . La gendarmerie présente ainsi le cas typique d'une organisation capable très rapidement de mobiliser l'institution sur des projets d'action développés au niveau local.

31. . Les polices des Länder frontaliers et du service fédéral de protection des frontières.

32. . En particulier sur la base de l'art. 39-1 et 39-2 CAAS.

33. . Ainsi, à la réunion franco-allemande annuelle entre parquets généraux de la zone frontalière en 2002, le nouveau procureur général de Colmar a annoncé une politique de coordination renforcée entre les décisions des parquets portant sur des opérations de coopération transfrontalière.

34. . A l'exception de la gendarmerie, tous les services de police disposent avant le CCPD de structures anciennes de coopération internationale, à un niveau central et dans la zone frontalière.

35. . La gendarmerie et la police nationales disposent sur ce point du réseau des bureaux SIRENE qui assurent l'obligation, pour certaines opérations transfrontalières, de passer par le niveau central.

36. . Assistance administrative mutuelle internationale, au sein de la Direction nationale du renseignement et des enquêtes douanières. 
37. . Bien que la douane française et la douane allemande y aient des personnels.

38. . Au sein du SRPJ de Strasbourg et des Bureaux de police judiciaire des Länder, mais cela concerne aussi les commissariats binationaux gérés par la police aux frontières et le service fédéral de protection des frontières.

39. . Ce texte précise en effet que les structures de coopération frontalière qui reposent sur l'accord précédent de 1977 perdent leur validité et sont remplacées par le CCPD.

40. . Entretien avec un policier français au Centre de coopération policière et douanière à Offenbourg, le 8 novembre 1999.

41. . Les centres de formation linguistique créés entre la gendarmerie et la police du Bade-Wurtemberg à Lahr, puis à Müllheim ont permis de déconcentrer l'enseignement de l'allemand dispensé à l'EIREL sur des structures binationales créées à la frontière, et ainsi de libérer au sein de l'EIREL des capacités d'enseignement pour d'autres langues. 42. . L'incrimination de " recel de cadavre » dans la législation française, à l'inverse de l'Allemagne, interdit en effet l'utilisation de cadavres humains pour la formation canine. Les chiens sont donc formés moins efficacement.

43. . Cette nécessité est ainsi satisfaite par exemple dans la construction du nouvel hôtel de police à Strasbourg. Ce bâtiment, qui regroupe à présent l'ensemble des services de la police nationale de Strasbourg, et inauguré en 2002, retient pour le service de coopération propre au SRPJ de Strasbourg un espace de bureaux et une salle de réunion capable d'accueillir les représentants des nombreux partenaires d'outreRhin.

44. . Cette argumentation est ainsi à la base de la fourniture d'un bateau neuf à la Compagnie fluviale de gendarmerie du Rhin à la fin des années 1990. Ce moyen de pression ne fonctionne cependant que lorsque l'institution est en mesure de suivre précisément les enjeux locaux. La capacité du local à gagner des ressources supplémentaires est ainsi beaucoup plus réduite dans le cas des services locaux de la police aux frontières.

45. . Par exemple, par l'abandon progressif par les services allemands des mesures de poursuite transfrontalière.

46. . Gérés à la frontière par la police aux frontières et le service fédéral de protection des frontières.

47. . Les services allemands demandaient en particulier la mise en place d'une permanence de nuit du côté français.

48. . Article 41-4, a et b de la CAAS.

49. . En interne, les services responsables de la coordination des opérations

transfrontalières recommandent même à présent à leurs services opérationnels

frontaliers de renoncer globalement à l'usage de la mesure de poursuite

transfrontalière vers la France.

50. . La mise en place d'autres centres de coopération policière et douanière est en effet en cours ou partiellement achevée aux frontières italienne, espagnole, belge et suisse de la France. 


\section{RÉSUMÉS}

La coopération policière et douanière en Europe repose sur des instruments juridiques multilatéraux et bilatéraux. Les plus récents (la convention d'application des accords de Schengen et l'accord franco-allemand de Mondorf-les-bains de 1997, puis les divers autres accords bilatéraux élaborés sur le modèle de ce dernier) ont fait de la coopération policière dans les zones frontalières des Etats l'espace d'interactions intenses entre un grand nombre d'acteurs et de systèmes de contrôle nationaux. Dans cette confrontation quotidienne, les transactions professionnelles entre acteurs policiers étrangers travaillent à modifier les rôles, mais aussi les modes et les priorités de l'action des services locaux et centraux. L'analyse détaillée des influences croisées des règles de droit et des paramètres de l'action policière à la frontière franco-allemande permet d'esquisser des hypothèses sur les transformations en cours dans le système national français de sécurité.

The European police and custom cooperation is based on multilateral and bilateral legal tools. The most recent ones (the convention for implementation of the Schengen agreements, the 1997 German-French agreement of Mondorf-les-bains, and the further bilateral agreements made on the base of the latter) have made out of the police cooperation in internal border regions a place of very intense interactions between a high number of national actors and control systems. In this daily face-to-face, the professional transactions between foreign police actors tend to modify roles as well as ways and priorities of intervention for regional and central police services. The detailed analysis of cross-influences of laws and parameters of police action at the GermanFrench border allows drafting some hypotheses on the transformations underway in the French national security system.

\section{INDEX}

Index géographique : Allemagne, France

Mots-clés : coopération internationale, police, frontières 